\title{
Ketolide agents HMR 3004 and HMR 3647 (telithromycin) inhibit the growth of Plasmodium falciparum in vitro
}

\author{
Marema Makgatho ${ }^{1}$, Eric Maimela ${ }^{2}$, Felix Mbajiorgu ${ }^{3}$
}

1. Department of Medical Sciences, Public Health, Health Promotion, Faculty of Health Sciences, University of Limpopo, South Africa.

2. Disease Surveillance and Epidemiology Unit, Provincial Department of Health, Limpopo Province, South Africa.

3. School of Anatomical Sciences, University of Witswatersrand (Wits), South Africa.

\begin{abstract}
Background: Malaria is on the increase due to emergence of parasite drug resistance and there is thus an urgent need for the development of new antiparasitic drugs effective at low concentrations. Ketolides antibiotics are used for treatment of various ailments and are relevant candidates to establish antiparasitic activity.

Objectives: The present study investigates the activity of ketolide compounds HMR 3004 and HMR 3647 (telithromycin) (0.025-12.5 $\mu \mathrm{M}$ ) for activity against chloroquine-sensitive and resistant strains of Plasmodium falciparum in vitro.

Methods: The antiplasmodial activity of the two ketolide agents were determined using microscopic and colorimetric [lactate dehydrogenase assay] procedures.

Results: Both HMR 3004 and HMR 3647 caused a dose-dependent inhibition of growth of both parasite strains with IC50 values 3 and $15 \mathrm{nM}$, respectively. Suppression of parasite growth was evident after 8 hours of exposure to both agents at $12.5 \mu \mathrm{M}$ with total parasite clearance achieved at 40 hours.

Conclusion: The results indicate lack of cross-resistance between the ketolide compounds and chloroquine, implying presence of a drug target different from that of chloroquine. The particular drug target has still to be investigated but the stage-specific results indicate that it is expressed in all parasite growth phases. These observations demonstrate the anti-malarial potential of the ketolide antimicrobial agents.

Key words: Chloroquine, ketolides, macrolides, malaria, Plasmodium falciparum.

DOI: http://dx.doi.org/10.4314/ahs.v15i4.28

Cite as: Makgatho M, Maimela E, Mbajiorgu F. Ketolide agents HMR 3004 and HMR 3647 (telithromycin) inbibit the growth of Plasmodium falciparum in vitro. Afri Health Sci. 2015;15(4):1271-6. http:// dx.doi.org/10.4314/abs.v15i4.28
\end{abstract}

\section{Introduction}

According to the World Health Organization report, 207 million new malaria cases were recorded in $2012^{1}$. There has since been a observable decline in malaria cases that is attributable to proper intervention strategies like introduction of long-lasting insecticidal nets

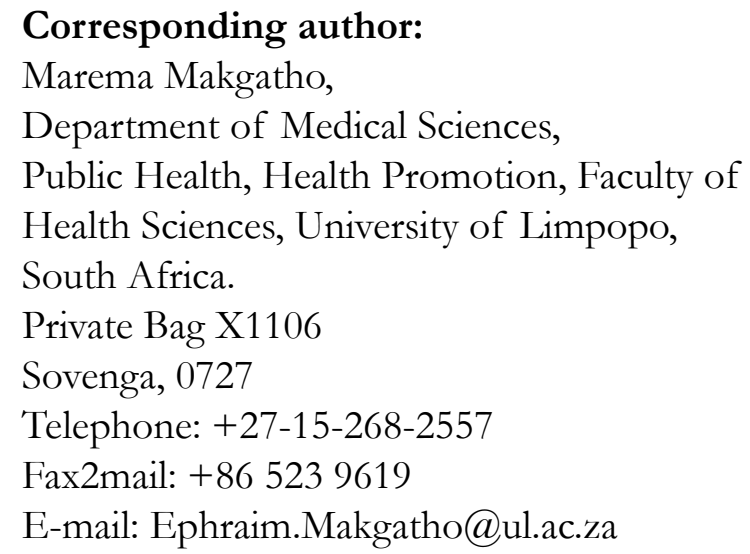

(LLINs) and artemisinin-based combination therapy $(\mathrm{ACT})^{2,3,4,5}$. The relevance of use of ACT is currently challenged by recent reports of reduced susceptibility and resistance in South-East Asia ${ }^{6,7}$. Drug development efforts are focused on the design of compounds which have reduced toxicity and increased potency, as well as on the development of agents which potentiate the activities of existing anti-malarial agents ${ }^{8}$. An alternative approach has been to examine the antiplasmodial potential of existing anti-bacterial agents ${ }^{9,10}$ and the riminophenazines that have shown promise ${ }^{11}$. Presently, the World Health Organization (WHO) prefers use of artemisin in-based combination therapy (ACT) and hybrid molecules for tailored delayed acquisition of resistance and/or curtailing it in totality ${ }^{12,13}$.

Structural modification of the prototype, 14-membered macrolide, erythromycin A, has led to the development of a novel class of macrolide agents, the ketolides. The major alteration characterising ketolides is 
replacement of the 3-cladinose of the macrolide ring by a keto-group. Ketolides inhibit microbial growth by interfering with the translation of messenger RNA, and exhibit increased potency, broadened antimicrobial spectrum and impressive accumulation in tissues and blood $^{14,15,16,17}$.
Although the improved anti-bacterial properties of the ketolides are well-recognised and characterised, little or no work appears to have been conducted on the anti-malarial potential of these agents. In the current study, we have investigated the effects of HMR 3004 and HMR 3647 (Figure 1), which have a carbamate group at $\mathrm{C} 11 / \mathrm{C} 12$ of the lactone ring with alkyl-aryl extensions on the growth of Plasmodium falciparum in vitro.

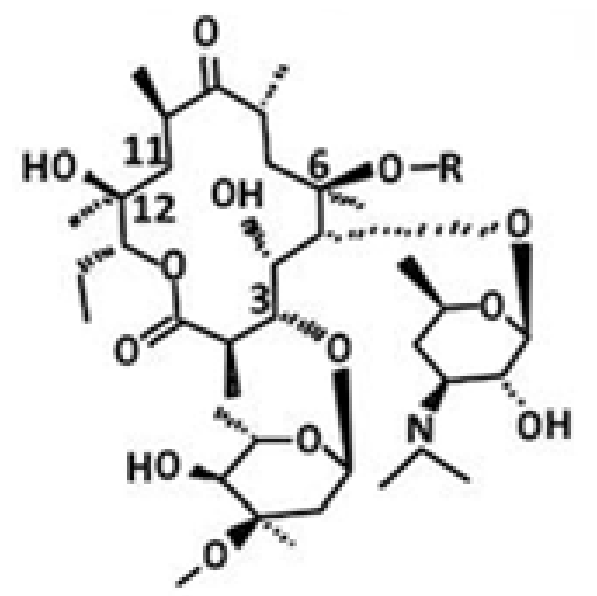

\section{Erythromycin A/Clarithromycin}

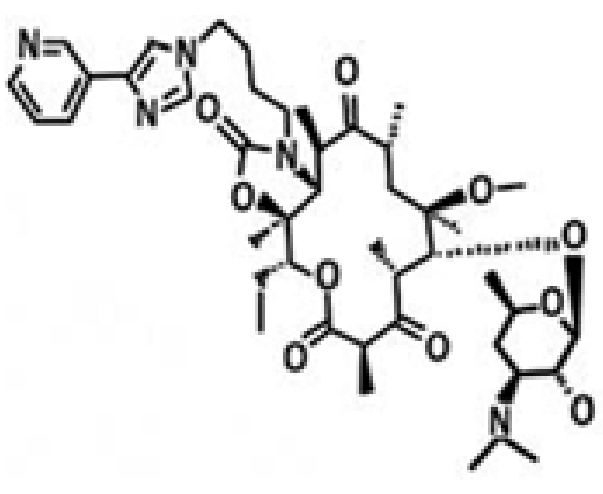

Telithromycin (HMR 3647)

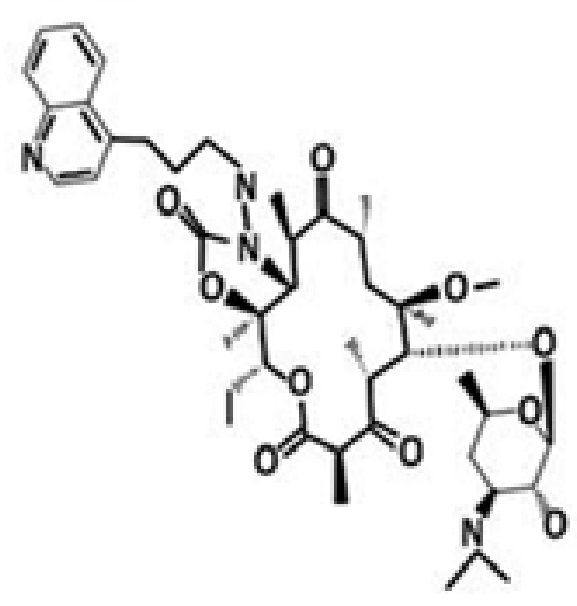

HMR 3004

\section{Figure 1: Structures of erythromycin A, telithromycin (HMR 3647) and HMR 3004.}

\section{Materials and methods}

\section{Parasites strains and culturing conditions.}

The chloroquine-resistant (FCR-3) and sensitive (FAB9) strains of P. falciparum were maintained in a continuous culture system of human erythrocytes (blood group $\mathrm{O}+$ obtained from the SA Blood Transfusion
Services) in RPMI 1640 culture medium supplemented with $2 \mathrm{mM}$ L-glutamine, $25 \mathrm{mM}$ HEPES, $25 \mathrm{mM} \mathrm{NaH}$ CO3 and 10\% human $\mathrm{AB}+$ serum. The suspension was maintained at $37^{\circ} \mathrm{C}$ in an atmosphere of $5 \% \mathrm{O}_{2} /$ $5 \% \mathrm{CO}_{2} / 90 \% \mathrm{~N}_{2}{ }^{10}$. Cultures were monitored daily by microscopic analysis of Giemsa-stained blood smears. 
Parasites cultures were synchronised to the ring stage by treatment with $5 \%$ D-sorbitol ${ }^{10}$.

\section{Antimicrobial agents.}

HMR 3004 and HMR 3647 were provided by Prof. RA Anderson (Department of Immunology, University of Pretoria, South Africa) and dissolved in $0.1 \mathrm{~N} \mathrm{HCl}$ to give a stock solution of $5 \mathrm{~g} / \mathrm{l}$ and further diluted in parasite culture medium.

\section{In vitro assays.}

For these assays, $20 \mu \mathrm{l}$ of infected erythrocyte suspension $(2 \%$ parasitemia and $0.5 \%$ final haematocrit) were exposed to $20 \mu \mathrm{l}$ of the ketolide compounds (0.025$12.5 \mu \mathrm{M})$. Parasites $(20 \mu \mathrm{l})$ in culture medium $(80 \mu \mathrm{l})$ and culture medium alone $(200 \mu \mathrm{l})$ served as positive and negative controls respectively. Solvent controls were also run. After $48 \mathrm{~h}$ incubation at $37^{\circ} \mathrm{C}$, parasite growth was determined by microscopic evaluation of Giemsa-stained slides and by use of the LDH assay ${ }^{18}$. For the LDH assay, $20 \mu \mathrm{l}$ of the parasite culture suspension was added to $100 \mu \mathrm{l}$ of the Malstat reagent and colour change measured at $620 \mathrm{~nm}$ in a microplate spectrophotometer. The FCR-3 (chloroquine-resistant) laboratory strain of P. falciparum was used for the stage-specific assays. Synchronised ring-stage parasite culture suspensions ( $2 \%$ parasitemia and $0.5 \%$ final haematocrit) were incubated with antimicrobial agents $(12.5 \mu \mathrm{M})$ and parasite development monitored at $8 \mathrm{~h}$ intervals for 48 hours using microscopy and colorimetry.

\section{Statistical analysis}

Results are expressed as the mean value \pm SEM for four experiments conducted in triplicate. The antiplasmodial activity of the compounds was expressed as percentage parasite growth and the IC50 values calculated using a Graph Pad Prism software version 5.00. Levels of statistical significance were calculated using Students t test (paired statistics), $\mathrm{p} \leq 0.05$ was considered significant.

\section{Results}

The effects of the test agents on the growth of the chloroquine-sensitive and resistant strains of P. falciparum as assessed by the colorimetric procedure are shown as IC50 values in Table 1. HMR 3004 and HMR 3647 exhibited a dose-related antiplasmodial activity $(\mathrm{p} \leq 0.05)$ comparable to that of chloroquine. HMR 3004 and HMR 3647 were also evaluated at $12.5 \mu \mathrm{M}$ for in vitro stage-specific activity and the results are shown in Figure 2. After an $8 \mathrm{~h}$ incubation period, parasite growth was suppressed by more than $50 \%$ with extended treatment leading to complete inhibition of parasite growth at around 35 hours with both antimicrobial agents, indicating a time dependent effect. These observations were confirmed by microscopy (data not shown).

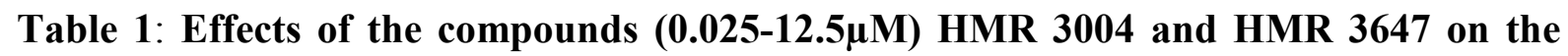
growth of the FAB-9 (chloroquine-sensitive) and FCR-3 (chloroquine-resistant) laboratory strains of $\boldsymbol{P}$. falciparum. Data are expressed as mean IC50 values \pm SEMs of four repeat experiments conducted in triplicates. Results on the activity of chloroquine not shown.

\begin{tabular}{lccc} 
& \multicolumn{3}{c}{ IC $_{50}(\mathrm{nM}) \pm$ SEM } \\
\cline { 2 - 4 } Strain & CQ & HMR 3004 & HMR 3647 \\
\hline FAB-9 & $\mathbf{3 1}$ & $\mathbf{3} \pm \mathbf{0 . 9}$ & $\mathbf{7} 0 \mathbf{0 . 1 2}$ \\
FCR-3 & $\mathbf{1 2 5}$ & $\mathbf{9} \pm \mathbf{2 . 1}$ & $\mathbf{1 5} \pm \mathbf{1 . 3 4}$ \\
\hline
\end{tabular}




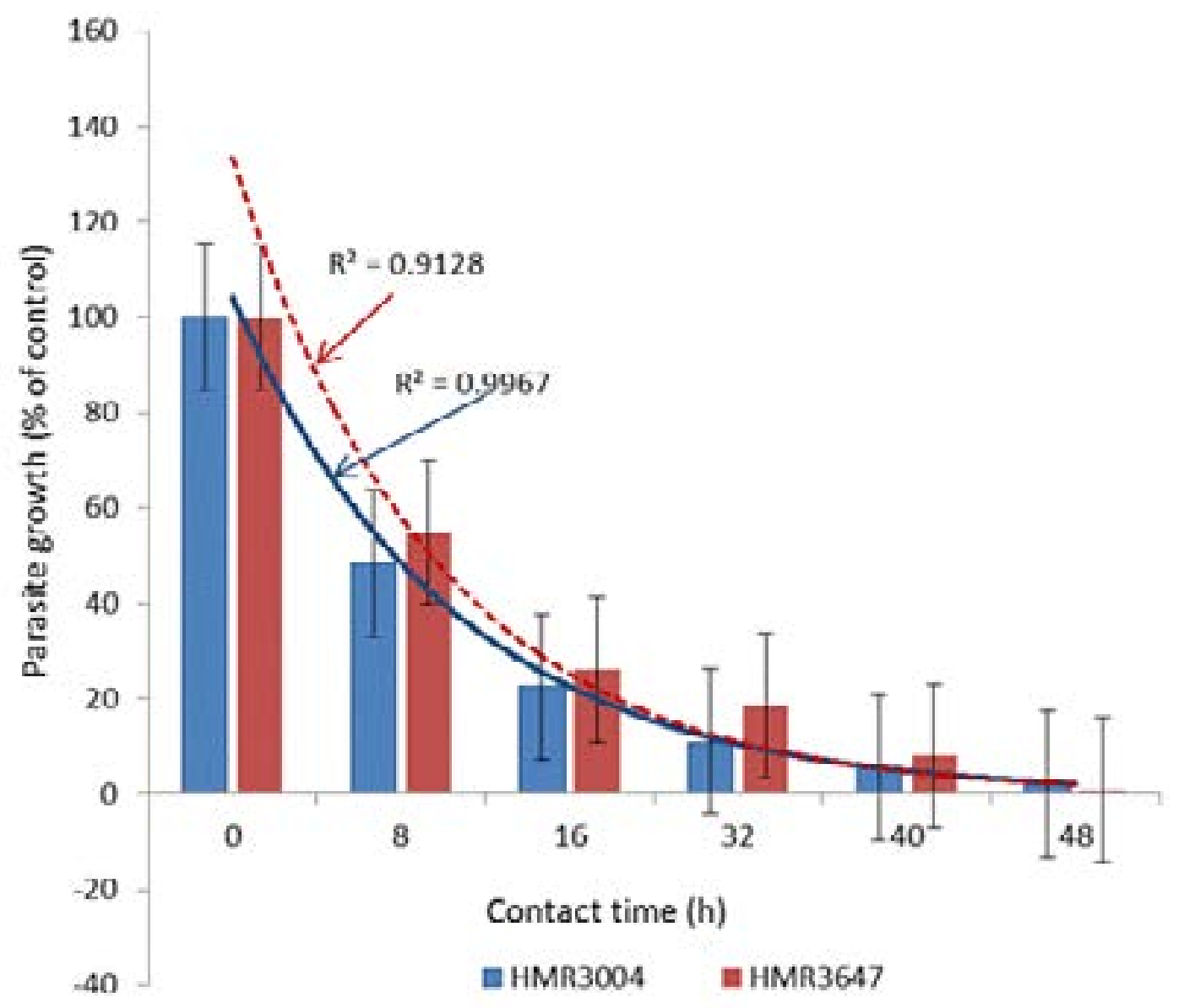

Figure 2: Stage-specific antimalarial activity of ketolide compounds HMR 3004 and HMR 3647 (12.5\& PubMed micro;M) on the FCR-3 laboratory strain of P. falciparum. Data from four repeat experiments with triplicate determinations in each are expressed as the mean percentage parasitemia of the control system \pm SEMs. $R^{2}=$ coefficient of determination.

\section{Discussion}

The results of the present study indicates that the ketolide compounds HMR 3004 and HMR 3647 inhibit the growth of $\mathrm{P}$. falciparum in vitro at concentrations probably comparable to those of chloroquine. Importantly, chloroquine-sensitive and resistant strains of the parasite were equally affected by the ketolides, demonstrating that the mechanisms of antiplasmodial action of these agents is likely to be distinct from that of chloroquine.

Antibiotics have been screened previously for in vitro antiplasmodial activity ${ }^{19,20,21,22}$. Of interest in one of the publications is the laboratory evaluation of macrolide erythromycin and azithromycin for activity against malaria parasites in combination with classical antimalarials. This study did show synergistic and additive activities of drug interactions ${ }^{20}$. Our study unfortunately did not explore drug combination activities in vitro. Telithromycin was also assayed in both laboratory and a mice models of malaria infection and did show activities that are not comparable to that in our present study $^{23,24}$. The differences in the results might be due to employment of different experimental platforms and parasite strains. For example, the kinetics model extends to over 48 hours thus including the re-infection stage, which is dissimilar to the same assay in the present study. The efforts to develop macrolides as antimalarials have proved fruitless.

HMR 3004 and HMR 3647 also inhibited the growth of the parasite increasingly with time, it does suggest that these chemicals inhibited parasite growth in a time-dependent manner. The observation may indicate that the molecular targets of these agents are expressed by the parasite and are affected as the parasite is exposed over time.

Ketolides inhibit bacterial growth by interfering with its ribosomal translation processes ${ }^{14}$. Plasmodium spp. have also been shown to express two forms of riboso- 
mal RNA, A- and S-type RNA found in the asexual and sexual forms of the parasite respectively ${ }^{17}$. Although these are potential targets of the ketolides in the parasite, existence of alternative or additional targets cannot be excluded.

\section{Conclusion}

The ketolide antimicrobials HMR 3004 and HMR 3647 possess antiparasite activity in vitro, and should further be evaluated with respect to molecular/biochemical mechanisms of action and efficacy in animal models of experimental chemotherapy.

\section{Acknowledgements}

The FAB-9 and FCR-3 laboratory strains of Plasmodium falciparum were kindly provided by Profs. Abram Louw (Department of Biochemistry, University of Pretoria, South Africa) and Robyn van Zyl (School of Therapeutic Sciences, University Witswatersrand, South Africa). The chemical entities were kindly provided by Prof Ronald Anderson (MRC Unit for Inflammation and Immunity, Tshwane National Health Laboratory Services and the University of Pretoria, South Africa). The laboratory work was financially supported by the South African Medical Research Council.

\section{Transparency declarations}

None to declare.

\section{References}

1. WHO. World Malaria Report 2013. Geneva, Switzerland.

2. Wongsrichanalai $\mathrm{C}$, Sibley $\mathrm{CH}$. Fighting drug-resistant Plasmodium falciparum: the challenge of artemisinin resistance. Clinical Microbiology and Infection, 2013; 14908-916.

3. WHO. World Malaria Report 2014. Geneva, Switzerland.

4. Zarocostas J. Malaria death falls by $47 \%$ bglobally between 2000 and 2013, WHO says. The Pharmaceutical Journal, 2014; 293, online| URI: 20067360

5. Mita T, Tanabe K. Evolution of Plasmodium falciparum drug resistance: implications for the development and containment of artemisinin resistance. Japanese Journal of Infectious diseases, 2012; 65:465-475.

6. WHO Global Malaria Programme.Update on artemisinin resistance - April 2012. Available from: http://www.who.int/malaria/publications/atoz/arupdate042012.pdf.

7. Muregi FW, Ishih A. Next generation antimalarial drugs: hybrid molecules as a new strategy in drug de- sign. Drug Devevelopment. Research, 2010; 71: 20-31. 8. Dahl EL, Rosenthal PJ. Multiple antibiotics exert delayed effects against the Plasmodium falciparum apicoplast. Antimicrobial Agents and Chemotherapy 2007; 51: 3485-3490.

9. Goodman CD, Su V, McFadden GI. The effects of anti-bacterials on the malaria parasite Plasmodium falciparum. Molecular and Biochemical Parasitology, 2007; 152: 181-191.

10. Makgatho ME, Anderson R, O'Sullivan JF, Egan TJ, Freeze JA, Cornelius N, Van Rensburg CEJ. Tetramethylpiperidine-substituted phenazines as novel anti-plasmodial agents. Drug Development Research, 2000; 50: 195-202.

11. Maude RJ, Woodrow CJ, White LJ. The artemisinin antimalarials: preserving the "magic bullet. Drug Development Research, 2010; 71: 12-19.

12. Capela R, Oliveira R, Goncalves LM, Domingoes A, Gut J, Rosenthal PJ, Lopes F, Moreira R. Artemisinin dipeptidyl vinyl sulfone hybrid molecules: design, synthesis, preliminary SAR for antiplasmodial activity and falcipain-2 inhibition. Bioorganic and Medicinal Chemistry Letters, 2099; 19: 3229-3232.

13. Lee Y, Choi JY, Fu H, Harvey C, Ravindran S, Roush WR, Boothroyd JC, Khosla C. Chemistry and biology of macrolide antiparasitic agents. Journal of Medicinal Chemistry, 2011; 54: 2792-2804.

14. Douthwaite S, Champney WS. Structures of ketolides and macrolides determine the mode of interaction with ribosomal target site. Journal of Antimicrobial agents and Chemotherapy, 2001; 48: 1-8 PubMed .

15. Sidhu AB, Sun Q, Nkrumah LJ, Dunne MW, Sacchettinni JC, Fidock DA. In vitro efficacy, resistance selection, and structural modelling studies implicate the malaria parasite apicoplast as the target of azithromycin. Journal of Biological Chemistry, 2007; 282: 2492-2504. 16. Vazifeh D, Abdelghaffar H, Labro TT. Cellular accumulation of the new ketolide RU64004 by human neutrophils: comparison with that of azithromycin and roxithromycin. Antimicrobial Agents and Chemotherapy, 1997; 41: 2099-2107.

17. McCutchan TF, Li J, McConkey GA, Rogers MJ, Waters UP. The cytoplasmic ribosomal RNAs of Plasmodium spp. Parasitology Today, 1995; 11: 134-138 PubMed .

18. Makler MT, Hinrichs DJ. Measurement of the lactate dehydrogenase activity of Plasmodium falciparum as an assessment of parasitemia. American Journal of Tropical Medicine and Hygiene, 1993; 48: 205-210.

19. Bowman JD, Merino EF, Brooks CF, Striepen B, Carler PR, Cassera MB. Antiaplicoplast and gameto- 
cydal screening to identify the mechanisms of action of compounds within the malaria Box. Antimicrobial Agents and Chemotherapy, 2014; 58:811-819.

20. Desgrouas C, Chapus C, Desplans J, Travaille C, Pascaul A, Baghdikian B, Ollivier E, Parzy D, Taudon $\mathrm{N}$, In vitro antiplasmodial activity of cepharathine. $M a$ laria Journal, 2014; 13:327-337.

21. In vitro and in vivo activity of Solithromycin (CEM101) against Plasmodium species. Antimicrobial Agents and Chemotherapy, 2012; 56:703-707.

22. Aminake MN, Schoof S, Sologub L, Leubner
M,Kirschner M, Arndt H, Pradel G. targeting parasite proteasome and apicoplast. Antimacrobial Agents and Chemotherapy, 2011; 55:1338-1348.

23. Nakornchai S, Konthiang P. Activity of azithromycin or erythromycin in combination with antimalarial drugs against multidrug-resistant Plasmodium falciparum in vitro. Acta Tropica, 2006; 100:185-191.

24. Barthel D, Schlitzer M, Pradel G. Telithromycin and Quinupristin-Dalfopristin induce delayed death in Plasmodium falciparum. Antimicrobial Agents and Chemotherapy, 2008; 52:774-777. 